\title{
X-ray Quasi-Lamellar Etched Multilayers: Analysis by Coupled-Mode Theory
}

\author{
Jean-Michel André, Karine Le Guen, and Philippe Jonnard \\ Laboratoire de Chimie Physique - Matière Rayonnement, UPMC Univ Paris 06, \\ CNRS UMR 7614, 11 rue Pierre et Marie Curie, F-75231 Paris cedex 05, France \\ ${ }^{*}$ Corresponding author: jean-michel.andre1@upmc.fr
}

\begin{abstract}
An etched multilayer (EM) is a two-dimensional structure obtained by etching a periodic multilayer following the profile of a grating. Generally a laminar grating shape, i.e. with a rectangular shape, is desired. However, we observe that different EM give different diffraction efficiency curves. We implement the coupled-mode theory to calculate the diffraction pattern of the EM as a function of the shape of the etching profile. Thus, we try to correlate the variability of the experimental reflectivity curves to various shapes of the profiles, rectangular and trapezoidal, and the corresponding simulated reflectivity curves.
\end{abstract}

Keywords: X-ray mirrors, Multilayers, Gratings, Reflectivity, Coupled-mode theory

Highlights: Coupled-mode theory is used to calculate the diffraction pattern of the etched multilayers and then compared to experiments. Reflectivity measurements show different curve shapes depending on the sample preparation. This is explained by the various shapes of the etching profile and partially confirmed by the calculations. 


\section{Introduction}

Multilayer gratings or etched multilayers (EMs), consisting in a periodic stack of bilayers with nanometric thickness laterally etched according to a periodic pattern, belong to this class of twodimensional photonic crystals that has opened up new ways for x-ray optics. Thus lamellar (or laminar) multilayer gratings can be used as efficient polychromators ${ }^{[1]}$ and are also implemented as improved monochromators with respect to mere multilayer Bragg mirrors for the soft-x-ray domain ${ }^{[2]-[8]}$. Recently, we have demonstrated that EMs can be used as dispersive element of curved-crystal soft x-ray spectrometer. In this case an improvement of the spectral resolution by a factor 4 was obtained in the $\mathrm{C}$ K $\alpha$ emission range ${ }^{[9]}$.

Calculations of the performances (efficiencies, band widths, ...) of these devices have been initially done in the framework of the "rigorous" modal theories which give rise to timeconsuming computations and tricky numerical problems ${ }^{[10]-[12]}$. Among the latter are the numerical instabilities ${ }^{[13][14]}$ and the mathematical difficulties in solving the transcendental equations to find the modal wavenumbers ${ }^{[15][16]}$. The level of difficulty is increasing as soon as the profile of the pattern is not lamellar, that is when the profile of the multilayer bar varies with the depth.

To overcome these difficulties, it has been recently proposed to appeal to the well-known coupled-waves approach; this latter has been successfully performed for the lamellar EMs ${ }^{[17]-}$ ${ }^{[20]}$. In this paper, we show that it is possible to implement the coupled-mode theory (CMT) to calculate the diffraction pattern of a quasi-lamellar grating with different profiles of the multilayer bar and we adopt this approach to study the influence of this profile. Quasi-lamellar means that the shape slightly differs from the rectangular profile: trapeze (bottom wider than top) and inverse trapeze shapes are considered. A series of EMs has been prepared from $\mathrm{Mo} / \mathrm{B}_{4} \mathrm{C}$ 
mirrors. Their diffraction pattern has been recorded around $182 \mathrm{eV}$ (corresponding to the $\mathrm{B} \mathrm{K}$ emission band) and compared to that originating from CMT calculations. The CMT approach has also been used in Ref. ${ }^{[18]}$ to consider deviation of the multilayer period as a function of the depth

and in Ref. ${ }^{[20]}$ to consider passivation and contamination layers, non-rectangular profiles and sidewall scalloping of the multilayer bar. Here, we show that the comparison between experimental and simulated reflectivity curves can help to understand how the profile shape influences the optical performances of etched multilayers.

\section{Description of the multilayer grating and geometry}

We consider the diffraction by an EM: the scheme of the grating and the geometry are given in Figure 1. The EM consists in a multilayer stack built up with $\mathrm{N}$ bilayers of thickness $d$; each bilayer is made up with a heavy material 1 of thickness $\mathrm{g} d$ and polarizability $\chi_{1}$, and a light material 2 of thickness $(1-\mathrm{g}) d$ and polarizability $\chi_{2}$. We consider abrupt interfaces so that the polarizability $\chi(z)$ of the bilayer is given by:

$$
\chi(z)=\chi_{1}+u(z)\left(\chi_{2}-\chi_{1}\right)
$$

where

$$
u(z)=\mathrm{g}+\sum_{t \neq 0} u_{t} \exp \left(\frac{2 i \pi t z}{d}\right)
$$

with

$$
u_{t}=\frac{(1-\exp (-2 i \pi t g))}{2 i \pi t}
$$

stands for the Heaviside unit step function. 


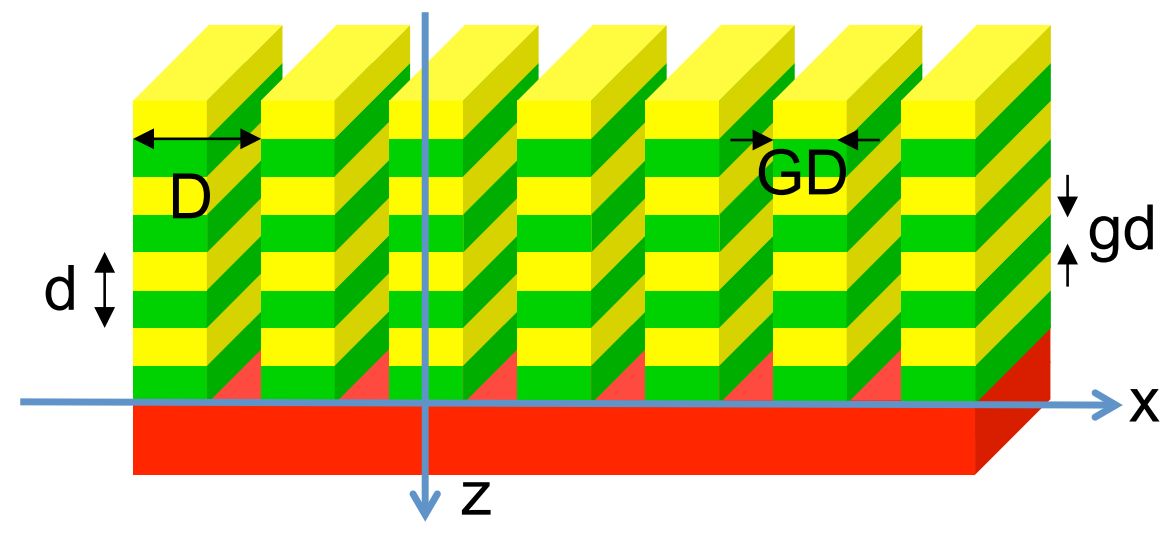

Fig. 1. Scheme of an EM with the multilayer bars having a rectangular profile (laminar grating). $d$ is the period of the multilayer stack while $D$ is the period of the grating. $g d$ is the thickness of the heavy material. $G(z) D$ is the width of the multilayer bar at the depth $z$, constant in this particular case. The crossing of the $x$ - and $z$-axes marks the origin $(x=0 ; z=0)$.

The stack is etched to obtain a grating of period $D$ with multilayer bar of width $G(z) D$ at the depth $z$. We will consider the case of grating with symmetrical shape with respect to the $z$ axis, the profile of which is given by:

$$
U(x, z)=G(z)+2 \sum_{t>0} \frac{\sin (\pi t \mathrm{G}(\mathrm{z}))}{\pi t} \cos \left(\frac{2 \pi t x}{D}\right)
$$

Then the function $G(z)$ allows us to deal with a grating whose bar profile is arbitrary. In the usual case of a laminar grating, one has $G(z)=\Gamma_{0}$. The two-dimensionnal polarizability of the grating under consideration is given by:

$$
\chi(x, z)=\chi(z) U(x, z)
$$

\section{Equations of coupled-mode theory}

We recall here the main equations developed on the basis of the CMT to solve our problem. More details are given in Ref. ${ }^{[19]}$. The electric field $E(x, z)$ in the structure obeys the following propagation equation for the $s$-polarization case: 


$$
\nabla^{2} E(x, y, z)+k^{2}(1-\chi(x, z)) E(x, y, z)=0
$$

solution of which can be expanded as:

$$
E(x, y, z)=\sum_{n=-\infty}^{+\infty} \varepsilon_{n}(z) \exp \left(i k_{\mathrm{x} n} x\right) \exp \left(i k_{y} y\right)
$$

with

$$
k_{x n}=k \cos \theta \cos \varphi+\frac{2 \pi n}{D}
$$

and

$$
k_{y}=k \cos \theta \sin \varphi
$$

where $k$ is the wavenumber in vacuum and $\theta$ and $\varphi$ the grazing and azimuthal angles of the incident radiation, respectively. Following the principle of the CMT, the $\mathrm{n}^{\text {th }}$ order of the field $\mathcal{E}_{n}(z)$ is searched as a superposition of two counter-propagating waves whose respective amplitudes $F$ and $B$ vary with $z$, that is:

$$
\mathcal{E}_{n}(z)=F_{n}(z) \exp \left(i k_{\perp n} z\right)+B_{n}(z) \exp \left(-i k_{\perp n} z\right)
$$

where

$$
k_{\perp n}=\sqrt{k^{2}-\left({k_{x n}}^{2}+{k_{y}}^{2}\right)}
$$

For the sake of uniqueness, the additional condition is required:

$$
\dot{F}_{n}(z) \exp \left(i k_{\perp n} z\right)+\dot{B}_{n}(z) \exp \left(-i k_{\perp n} z\right)=0
$$


where the dot indicates the derivative with respect to $z$. Substituting Eqs. (10) and (12) into Eqs. (6) and (7), it follows that the amplitudes satisfy the following system of differential equations:

$$
\begin{gathered}
\frac{d F_{n}(\mathrm{z})}{d z}=-\frac{i k^{2}}{2 k_{\perp n}} \chi(z) \sum_{m=-\infty}^{+\infty} U_{n-m}(z)\left(F_{n}(\mathrm{z}) \exp \left[+i\left(k_{\perp m}-k_{\perp n}\right) z\right]\right. \\
\left.+B_{n}(\mathrm{z}) \exp \left[-i\left(k_{\perp m}+k_{\perp n}\right) z\right]\right)
\end{gathered}
$$

In this study, we assume that there is a single plane wave incident on the grating, which gives the following boundary conditions:

$$
F_{n}(0)=\delta_{n, 0} \text { and } B_{n}(L)=0
$$

Let us note that this theory can be applied to the $p$-polarization case provided that one replaces in Eq. (6) the electric field by the modified magnetic field $\widetilde{H}(x, z)=H(x, z) / \sqrt{1-\chi(x, z)}$ and the polarizability $\chi(x, z)$ by the following modified susceptibility ${ }^{[17]}$ :

$$
\tilde{\chi}(x, z)=\chi(x, z)-\frac{1}{2 k^{2}} \frac{\nabla^{2} \chi(x, z)}{1-\chi(x, z)}+\frac{3}{4 k^{2}} \frac{[\vec{\nabla} \chi(x, z)]^{2}}{(1-\chi(x, z))^{2}}
$$

\section{Comparison with experiment}

The studied EM is designed as a narrow bandwidth monochromator for the spectral range around $180 \mathrm{eV}$ corresponding to the $\mathrm{B} \mathrm{K}$ emission band. It consists in $200 \mathrm{Mo} / \mathrm{B}_{4} \mathrm{C}$ bilayers deposited onto an ultra-smooth silicon substrate, then etched following a two main steps process: first deep UV photolithography; second fluorine reactive ion etching (RIE). The aimed grating period $D$ is $1 \mu \mathrm{m}$ and $\mathrm{G}$ ratio is 0.25 . The multilayer period $d$ and $g$ the ratio of the Mo thickness to this 
period are equal to $6.2 \mathrm{~nm}$ and 0.35 respectively. Thus the total thickness of the multilayer bar is $1240 \mathrm{~nm}$. The size of the samples is $10 \mathrm{~mm} \times 10 \mathrm{~mm}$.

The reflectivity of the multilayer (prior etching) has been measured at the $0.154 \mathrm{~nm}$ wavelength. The analysis of the reflectivity curve shows that the multilayer period is $6.11 \mathrm{~nm}$, the Mo thickness being $2.28 \mathrm{~nm}$ and the $\mathrm{B}_{4} \mathrm{C}$ one $3.83 \mathrm{~nm}$. The interfacial roughness is limited to $0.4 \mathrm{~nm} r m s$. Some fits have been done with the period thickness varying along the stack. In these cases, the maximum possible drift is $0.0002 \mathrm{~nm}$ per period, that is to say a difference of only $0.04 \mathrm{~nm}$ between the top and the bottom of the multilayer.

The diffraction efficiency, or reflectance, of the EM at the zero ${ }^{\text {th }}$ diffraction order of the grating and $1^{\text {st }}$ order of the multilayer has been measured at $182 \mathrm{eV}$ versus the glancing angle ${ }^{[9]}$ at the BEAR beamline of the ELETTRA synchrotron facility. The reflectivity curves of six different samples, a priori prepared in the same conditions but not in the same batch, are presented in Figure 2. They are shifted vertically for sake of clarity. A common general shape is observed: a main peak close to an angle of $35^{\circ}$ corresponding to the $1^{\text {st }}$ Bragg peak of the multilayer, surrounded by secondary peaks due to the coupling with the grating diffraction orders. However, all the curves are different: the maximum of reflectance varies between 0.075 and 0.150 ; the relative intensities of the secondary peaks change as well as their positions relative to the Bragg peak. For example, the intensity of the first feature toward the low angles varies from 0.034 to 0.054 and its position can move by about $0.1^{\circ}$, between 34.7 and $34.8^{\circ}$, leading this structure to be observed as a shoulder when too close from the Bragg peak (zero ${ }^{\text {th }}$ diffraction order of the grating). 


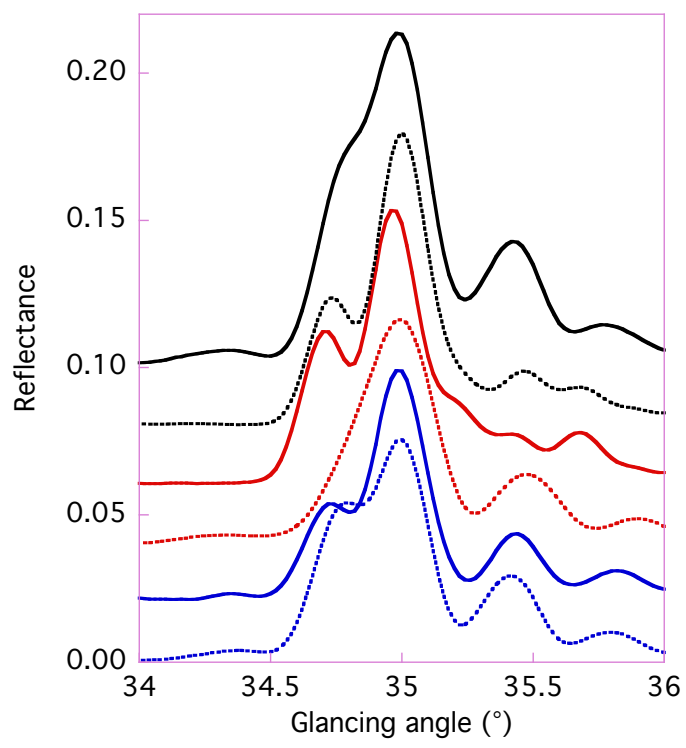

Fig. 2. Diffraction efficiency (reflectance) of six different etched multilayers. The curves are shifted vertically by 0.02 for sake of clarity.

It has been checked that the shape of the curves is the same at different location on the sample surface, that is to say the reproducibility problem is not due to a surface inhomogeneity introduced by the process of the multilayer. To gain in understanding this variability of the reflectivity curves, some samples have been cleaved in order to observe their cross section by scanning electron microscopy (SEM), see Figure 3. Let us note that the samples devoted to the SEM observation are not the one on which reflectivity measurements have been carried out.

First, it is observed that the grating period of $1.0 \mu \mathrm{m}$ is respected. However, depending on the sample, different shapes of the multilayer bars are observed. The grating is not perfectly laminar: multilayer bars can present the shape of a trapeze or some roundness. Thus the $G$ ratio value, even if close to 0.25 , can vary from 0.15 to 0.3 from the top to the bottom of the multilayer. Two origins of this problem, coming from the etching process itself have been identified: a bad contact between the mask and the substrate during the photolithography step; the difficulty to stop the RIE process just at the very border between the stack and the silicon substrate. Indeed, there is a thin $\mathrm{Cr}$ adhesion layer between the multilayer and the substrate, 
which is not sensitive to the etching process. Then, if the etching process is continuing after the layer is reached, ions will attack the foot of the multilayer bar and thus will lead to a nonrectangular shape.

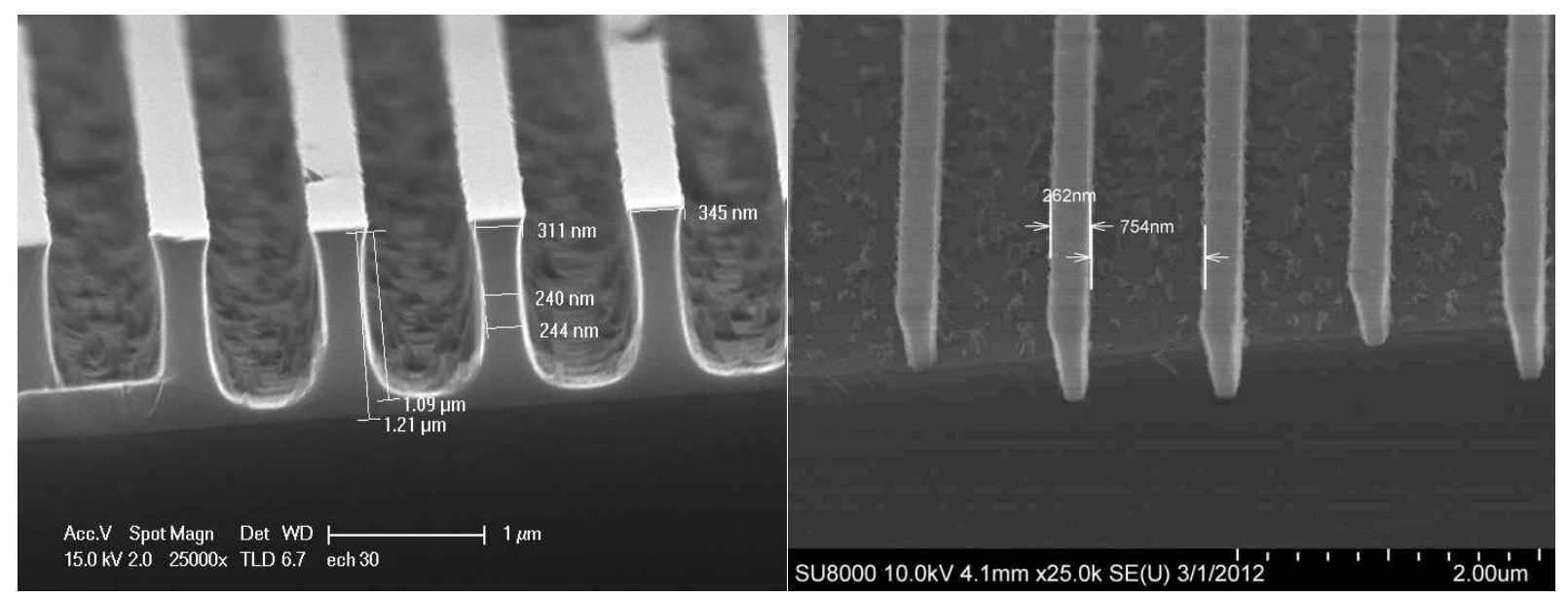

Fig. 3. Scanning electron microscopy images of cross sections of two different etched multilayers.

We believe that this variability of profile shapes can explain some part of the shape variations of the reflectivity curves. Thus, the developed CMT analysis is applied to study to what extent the profile of the multilayer bar influences the shape of the diffraction pattern of an EM. Five modes, i.e. 11 terms $(-5, \ldots, 0, \ldots,+5)$ were taken into account in the calculations. Figure 4 shows the EM efficiency in the range of the B K emission as a function of the glancing angle calculated for different profile shapes and for two mean $G$ ratios, 0.18 and 0.25 . In the first case the maximum of the simulated curves are normalized to the experimental reflectivity of one of the analysed samples. As explained above, the shape of the profile is taken into account through the depth variation of the $G$ ratio. The mathematical functions describing the considered shapes are mentioned below in the case of $G$ equal to 0.18 :

- $\quad$ rectangular (lamellar grating): $G(z)=0.18$;

- trapezoidal with the summit narrower than the basis: $G(z)=0.18+10^{-4} \mathrm{z}(\mathrm{z}$ in $\mathrm{nm})$;

- trapezoidal with the summit broader than the basis: $G(z)=0.18-10^{-4} \mathrm{z}(\mathrm{z}$ in $\mathrm{nm})$. 
These different shapes of the multilayer bars lead to small but sensitive reflectivity curve shapes. The maximum of reflectivity varies by 0.01 and the intensities of the secondary structures by 0.02 (about $30 \%$ relative variation) in the case of the secondary structure toward the high angles of the main peak.
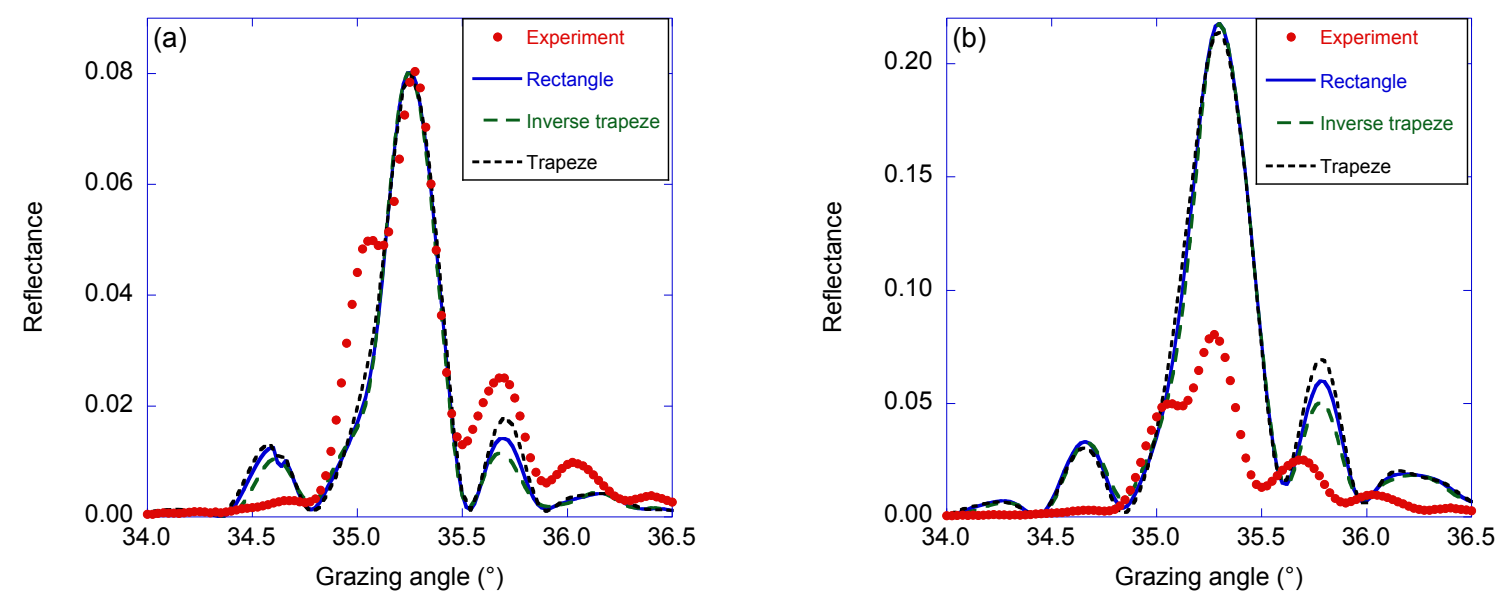

Fig. 4. CMT calculated reflectivity curves in the B K emission range for different profiles of the multilayer bars with the $G$ ratio of the multilayer bar width to the grating period equal to 0.18 (a) and 0.25 (b). The calculations with the $0.18 \mathrm{G}$ ratio have been normalized to the experimental reflectivity value.

The comparison of the experimental and calculated reflectivity curves shows that the reflectance is overestimated in the calculations by roughly a factor of 2.5, see Fig. 4(b) without normalization. A possible explanation can be the fact that the roughness at the multilayer interfaces as well as on the side of the multilayer bar is not taken into account. Now comparing the shape of the curves and the positions and relative intensities of the main and secondary maxima, it can be seen that none of the considered shapes is able to fully reproduce the experiment. For example the feature experimentally observed at $35.0^{\circ}$ is only a faint shoulder in the simulations. The trapeze shape with $G$ equal to 0.18 is the case where the position of the different structures is in better agreement with the experiment. In this case the intensity ranges from 0.011 to 0.018 in the simulations and is 0.025 experimentally. 
We have also made some calculations with an asymmetrical shape of the profile of the multilayer bar, that is to say a mix of rectangular shape for its ascending part (the part of the profile toward the lower $x$ ) and trapezoidal shape for its descending part (the part of the profile toward the higher $x$ ). In this case, the relative intensity of the shoulder at $35.0^{\circ}$ increases and the relative intensities of the secondary peaks changes more prominently with respect to the symmetrical shapes. However, this does not seem compatible with the rather symmetrical shapes observed on the SEM images, see Fig. 3.

\section{Conclusion}

We have implemented the coupled-mode theory to calculate the diffraction pattern of an etched multilayer as a function of the shape of the etching profile. The simulations are not able to fully reproduce the features of the experimental reflectivity curve, however the positions and relative intensities of the secondary structures due to the coupling with the grating diffraction orders are sensitive to the shape of the multilayer bar and also to the ratio of the multilayer bar width to the grating period. To improve the comparison between experiments and simulations it does not seem that considering a variation of the multilayer period along the surface could be considered because we have verify the reproducibility of the reflectivity measurements at different positions of the surface. A drift of periodicity along the stack could also be envisaged but is very limited in our case, $0.04 \mathrm{~nm}$ at maximum over 200

periods, which could lead to some broadening and some reflectivity loss ${ }^{[18]}$, and could explain a part of the observed discrepancy.

Experimentally, we observed in the boron $\mathrm{K}$ range that the reflectivity curves from different $\mathrm{Mo} / \mathrm{B}_{4} \mathrm{C}$ samples prepared a priori in the same conditions exhibit different shapes. 
This comes from small inhomogeneity from one sample to another during the preparation that leads to different shapes of the multilayer bars as shown by the SEM images.

\section{Acknowledgments}

Incoatec is thanked for providing us with the multilayers. J.-R. Coudevylle and N. Isac from IEF, Orsay, France, are thanked for their help during the etching process. F. Delmotte, from Institut d'Optique, Palaiseau, France, is thanked for performing the reflectivity experiment at $0.154 \mathrm{~nm}$ and its fit.

\section{References}

[1] K. Krastev, J.-M. André, R. Barchewitz, J. Opt. Soc. Am. A 1996, 13, 2027.

[2] A.-E. Sammar, J.-M. André, M. Ouahabi, B. Pardo, R. Barchewitz, C. R. Acad. Sci., Ser. 2, 1993, 316, 1055.

[3] A. Sammar, K. Krastev, J.-M. André, R. Barchewitz, R. Rivoira, Rev. Sci. Instrum. 1997, 68, 2969.

[4] J.-M. André, R. Benbalagh, R. Barchewitz, M.-F. Ravet, A. Raynal, F. Delmotte, F. Bridou, G. Julié, A. Bosseboeuf, R. Laval, G. Soullié, C. Rémond, M. Fialin, Appl. Opt. 2002, 41, 239.

[5] R. Benbalagh, J.-M. André, R. Barchewitz, P. Jonnard, G. Julié, L. Mollard, G. Rolland, C. Rémond, P. Troussel, R. Marmoret, E.O. Filatova, Nucl. Instrum. Methods Phys. Res. Sect. A 2005, 541, 590 .

[6] P. Jonnard, K. Le Guen, J.-M. André, X-Ray Spectrom. 2009, 38, 117.

[7] I.V. Kozhevnikov, R. van der Meer, H.M.J. Bastiaens, K.-J. Boller, F. Bijkerk, Opt. Express 2010, 18, 16234.

[8] R. van der Meer, B. Krishnan, I.V. Kozhevnikov, M.J. De Boer, B. Vratzov, H.M.J. Bastiaens, J. Huskens, W.G. van der Wiel, P.E. Hegeman, G.C.S. Brons, K.-J. Boller, F. Bijkerk, Proc. SPIE 2011, 8139, 81390Q.

[9] P. Jonnard, K. Le Guen, J.-M. André, J.-R. Coudevylle, N. Isac, X-Ray Spectrom. 2012, 41,308 .

[10] A. Sammar, J.-M. André, B. Pardo, Opt. Commun. 1991, 86, 245.

[11] A.I. Erko, B. Vidal, P. Vincent, Y.A. Agafonov, V.V. Martynov, D.V. Roschupkin, M. Brunel, Nucl. Instrum. Methods Phys. Res. Sect. A 1993, 333, 599.

[12] L. Li, J. Opt. Soc. Am. A 1993, 10, 2581.

[13] F. Montiel, M. Nevière, J. Opt. Soc. Am. A 1994, 11, 3241.

[14] K. Krastev, F. Le Guern, K. Coat, R. Barchewitz, J.-M. André, M.-F. Ravet, E. Cambril, F. Rousseaux, P. Davi, Nucl. Instrum. Methods Phys. Res. Sect. A 1996, 368, 533.

[15] L. Botten, M. Craig, R. McPhedran, J. Adams, J. Andrewartha, Opt. Acta 1981, 28, 1087.

[16] G. Tayeb, R. Petit, Opt. Acta 1984, 31, 1361.

[17] I.V. Kozhevnikov, R. van der Meer, H.M.J. Bastiaens, K.-J. Boller, F. Bijkerk, Opt. Express 2011, 19, 9172.

[18] R. van der Meer, I.V. Kozhevnikov, B. Krishnan, J. Huskens, P. Hegeman, C. Brons, B. 
Vratzov, B. Bastiaens, K. Boller, F. Bijkerk, AIP Advances 2013, 3, 012103.

[19] J.-M. André, P. Jonnard, K. Le Guen, UVX2012 2013, 02001.

[20] R. van der Meer, I.V. Kozhevnikov, H.M.J. Bastiaens, K.-J. Boller, F. Bijkerk, Opt. Express 2013, 21, 13105. 